nur die „strukturelle Ursprungsformel“ Zweiter Kammern, sondern auch die „funktionelle Schüsselformel“ für ihre Bedeutung im politischen Prozess. Für ihren Vergleich der Funktion unterschiedlich situierter Zweiter Kammern greifen Riescher und Ruß auf den Vetospieleransatz von George Tsebelis zurück und arbeiten die „funktionale Relevanz Zweiter Kammern zur Durchsetzung verhandlungsdemokratischer Mechanismen und zur Abschwächung des Mehrheitsprinzips“ heraus. Ihr Hinweis, dass es neben den normierten auch potentielle Funktionen sind, die einer Zweiten Kammer zur Akzentsetzung offen stehen (wie etwa die Herstellung von Gegenöffentlichkeit), erschließt eine grundsätzliche Aufgabe Zweiter Kammern, nämlich „Agenda-Setter zu kontrollieren“ (S. 514). Auf diese Weise könnten sie erstens bereits bei der Bestimmung von Themen ein Verhandlungselement einführen und es zweitens einem Agenda-Setter zumindest schwerer machen, mit seiner Formulierung eines Gesetzesvorhabens auch gleich die Antwort vorzustrukturieren. Doch selbst bei dieser bescheiden anmutenden Funktionszuweisung gibt es - so die Autorinnen - Zweite Kammern, die auch dann keine nennenswerten Legislativfunktionen aufzuweisen haben. Während früher auch das Japanische Oberhaus in diese Kategorie gehörte, konnte dieses in den letzten Jahren seine Position ausbauen. Damit findet sich inzwischen allein der Österreichische Bundesrat in der Kategorie einer Zweiten Kammer ohne erwähnenswerte Gesetzgebungsfunktion.

Es passt in das Gesamtbild, das die beiden Bände beim Leser hinterlassen, dass der analytische Gehalt des Schlusskapitels in dem von Riescher / Ruß / Haas herausgegebenen Handbuch deutlich weiter reicht als das Gegenstück von Sven Leunig. Zweifelsohne verspricht die Lektüre beider Bücher einen Gewinn; bei Ressourcenknappheit kann aber eine Präferenz ausgesprochen werden: „Zweite Kammern“ von Riescher / Ruß / Haas lohnt sich nicht nur für diejenigen, die ohnehin an bikameralen Systemen interessiert sind.

Ursula Münch

\title{
Landesverfassungsgerichte: Einfluss politologisch untersucht
}

Flick, Martina: Organstreitverfahren vor den Landesverfassungsgerichten. Eine politikwissenschaftliche Untersuchung, Peter Lang Verlag, Frankfurt am Main u.a. 2011, 303 Seiten, $€ 56,60$.

Martina Flick untersucht in ihrer Dissertation aus politikwissenschaftlicher Sicht den Einfluss der Landesverfassungsgerichte auf den politischen Prozess in den Ländern. Ihre Arbeit entstand im Rahmen des Forschungsprojekts „Demokratiemuster in den deutschen Bundesländern und ihre politischen, gesellschaftlichen und ökonomischen Auswirkungen “, das an der Universität Konstanz von den Professoren Adrian Vatter und Markus Freitag geleitet wurde; beide haben die Doktorarbeit auch betreut.

Es ist sehr zu begrüßen, dass sich die Politikwissenschaft der föderalen Vielfalt der politischen Systeme der Bundesländer widmet, um Gemeinsamkeiten und Unterschiede zu untersuchen sowie Akteure und ihre Beziehungen in den Blick zu nehmen. Im Hinblick auf den Einfluss der Landesverfassungsgerichte ist die Analyse der Organstreitverfahren ein viel versprechender Anknüpfungspunkt, denn es geht in Streitigkeiten zwischen obersten 
Landesorganen und Teilen von ihnen um ihre verfassungs- oder geschäftsordnungsmäßigen Rechte.

Flick weist zunächst darauf hin, dass sich der - historisch gewollte - politische Charakter der Verfassungsgerichtsbarkeit auf der institutionellen Ebene zum einen an dem hohen Anteil an nicht richterlichen Mitgliedern festmachen lässt (wobei deren tatsächlicher Einfluss gegenüber den juristisch geschulten Richtern eher gering sein dürfte), zum anderen an der Wahl der Richter durch die Landtage. Die Verfahrensvorschriften für die Organstreitverfahren in den Ländern haben sich im Laufe der Zeit weitgehend den für das Bundesverfassungsgericht geltenden Regeln angenähert, so dass sich die wenigen Unterschiede auf Häufigkeit und Ausgang wohl nicht auswirken dürften.

Sodann legt sich die Autorin - in Auseinandersetzung mit unterschiedlichen Forschungsansätzen, insbesondere aus den USA - auf ihre Prämissen fest. Sie betrachtet Verfassungsgerichte als strategisch handelnde Akteure, die in ihren Entscheidungen die Reaktionen anderer Akteure berücksichtigen, vor allem um die Akzeptanz ihrer Entscheidungen sicherzustellen. Sie trifft sich an diesem Punkt mit der juristischen Methodik der Verfassungsinterpretation, zu der auch die Analyse möglicher Entscheidungsfolgen im Hinblick auf den Sinn und Zweck der Norm gehört. Ferner geht sie aus von einem „akteurszentrierten Institutionalismus“, demzufolge Handlungsorientierungen abhängig sind von institutionellen und kontextunabhängigen Einflüssen auf die Akteure und ihrer Interaktion.

Flick spezifiziert anschließend ihr Untersuchungsziel durch drei Unterfragen. Als erstes stellt sie fest, dass Organklagen zu 93 Prozent von der Opposition erhoben werden, und fragt nach den Faktoren, die diese Häufigkeit beeinflussen. Dabei arbeitet sie mit statistischen Auswertungen. Sie kann nachweisen, dass insbesondere die politische Ausrichtung der Opposition Auswirkungen auf die Klagehäufigkeit hat. Fraktionen von den Rändern des politischen Spektrums sind signifikant häufiger beteiligt an Organstreit-Verfahren, weil sie - so vermutet Flick - ohnehin weniger auf Sacharbeit im Detail aus sind als auf spektakuläre Aktionen in der Öffentlichkeit (man könnte auch sagen, auf Klamauk). Ein anderer Grund könnte sein, dass sie gerade deshalb von den anderen Fraktionen häufiger in ihren parlamentarischen Wirkungsmöglichkeiten begrenzt werden.

Einen gewissen Einfluss auf die Klagehäufigkeit scheint auch das Ausmaß der kodifizierten Kontrollrechte der Opposition zu haben. Das erscheint plausibel, denn je klarer diese Rechte geregelt sind, desto weniger ist die Opposition auf die Klärung im Organstreitverfahren angewiesen.

Dass sich der Einfluss der Opposition auf die Besetzung der Verfassungsgerichte sowie die Klagehäufigkeit auswirkt, kann Flick ebenso wenig nachweisen wie eine Abhängigkeit der Klagehäufigkeit von der Stärke der Regierungsfraktionen, etwa im Falle einer Großen Koalition.

Über die von Flick erwogenen Faktoren hinaus kommen weitere in Betracht: Die Rücksicht auf potentielle Koalitionspartner könnte zur Zurückhaltung bei Organklagen beitragen - sie kann sich sowohl in der Wahrung der Kontrollrechte von Seiten der Mehrheit auswirken als auch auf das Klageverhalten der Opposition. Der Ausgang früherer Verfahren kann die Bereitschaft zur Klage ebenfalls beeinflussen: Obsiegen reizt zu weiteren Verfahren, Niederlagen führen zur Zurückhaltung. Daneben können andere Instanzen der Mediation eine Rolle spielen, zum Beispiel unparteiisch arbeitende wissenschaftliche Dienste der Parlamente, die umstrittene Fragen der Kontrollrechte im Vorfeld von gerichtlichen Verfah- 
ren klären; sie können dann streitvermeidend wirken, wenn die Beteiligten ihre Ergebnisse akzeptieren.

Zum zweiten sucht Flick nach Faktoren für die Erfolgsaussichten der Opposition bei Organstreitverfahren vor den Landesverfassungsgerichten.

Einen Zusammenhang zwischen der (parteipolitisch beeinflussten) Zusammensetzung der Verfassungsgerichte und der Erfolgsaussicht kann Flick mit rein quantitativen, statistischen Methoden nicht nachweisen, auch nicht am konkreten Beispiel des Berliner Verfassungsgerichtshofs. Dass ihr ein solcher Nachweis nicht gelingt, ist sicherlich ein Beleg für die Unabhängigkeit der Richterpersönlichkeiten und die Eigenständigkeit juristischer Argumentation, die offenbar doch mehr ist als Vehikel zur Begründung ideologisch gewollter Entscheidungen.

Ungeachtet von parteipolitischen Überlegungen bei der Besetzung der Richterstellen können natürlich einzelne Richterpersönlichkeiten die Linie eines Gerichts beeinflussen oder die Berichterstatter mit ihrer politischen, religiösen, aber auch juristisch-wissenschaftlichen Ausrichtung einzelne Entscheidungen. Das ist aber in der Tat durch statistische Vergleiche nicht zu ermitteln und auch nicht generell ein Faktor, der sich auf die Erfolgsaussichten der Opposition auswirkt. Ein weiterer juristischer, aber politologisch kaum fassbarer Faktor für die Erfolgsaussichten einer Organklage ist die Qualität der juristischen Argumentation - eine penible Aufarbeitung des Sachverhalts und eine qualifizierte juristische Argumentation erhöhen die Chancen auf einen positiven Ausgang des Verfahrens.

Als dritten und letzten Punkt untersucht Flick den Einfluss der Landesverfassungsgerichte auf das Parlamentsrecht in den Bundesländern und hier insbesondere auf die Rechte der parlamentarischen Minderheiten. Sie kommt zu dem Ergebnis, dass Landesverfassungsgerichte zwar häufiger über parlamentsrechtliche Fragen entscheiden als das Bundesverfassungsgericht. Dass sie dabei die Minderheitsrechte ausbauen, lässt sich aber - von wenigen Ausnahmen abgesehen - nicht belegen. Entscheidend dafür seien vielmehr die einschlägigen Entscheidungen des Bundesverfassungsgerichts, die Vorbildfunktion einzelner Landesverfassungen, insbesondere das der 1990 reformierten schleswig-holsteinischen Verfassung, sowie die Empfehlungen der Konferenz der Landtagspräsidenten zum Ausbau der parlamentarischen Kontrollrechte.

Insgesamt bietet die Arbeit einen guten Überblick und Vergleich der Verfassungsgerichtsbarkeit der Länder und die Auswirkung ihrer Rechtsprechung in Organstreitverfahren. Flick schöpft die wissenschaftliche Erkenntnisfähigkeit quantitativer, statistischer Verfahren zum Thema aus. Zu ihren Ergebnissen gehört, dass weitere Erkenntnisse nur mit qualitativen Methoden erreichbar sind, etwa durch in die Tiefe gehende Fallstudien. Der Rezensent rät dabei zur Kooperation von Politik- und Rechtswissenschaftlern, um alle also auch rechtswissenschaftliche - Erkenntnisquellen zu erschließen. Dass Flick eine der Entscheidungsbesprechungen des Rezensenten (der Jurist ist) zur politikwissenschaftlichen Forschung zählt, ist ein verzeihlicher und für den Rezensenten ehrenhafter Irrtum. Kein inhaltlicher, sondern formaler Kritikpunkt ist das Satzbild zahlreicher Tabellen, deren Text auf dem Kopf steht. Hier zeigt sich wieder einmal der ärgerliche Mangel einer wenigstens groben Lektorierung von Dissertationen. 